\title{
SAFE DESIGN OF COOLED TUBULAR REACTORS FOR EXOTHERMIC, MULTIPLE REACTIONS. CONSECUTIVE REACTIONS
}

\author{
K. R. WESTERTERP ${ }^{\dagger}$ and R. R. M. OVERTOOM \\ Chemical Reactions Engineering Laboratories, Department of Chemical Engineering, Twente University of \\ Technology, PO Box 217, 7500 AE Enschede, The Netherlands
}

(Received 27 November 1983; in revised form 17 April 1984; accepted 14 May 1984)

\begin{abstract}
The model of the pseudo-homogeneous, one-dimensional, cooled tubular reactor is applied to two consecutive, irreversible first order reactions. A criterion is derived to obtain a desired integral yield. Based on this criterion three requirements are formulated, which enable us to choose the relevant design and operating conditions. If any of the requirements are met, the reactor is also safe with respect to runaway. In an illustration the results are applied to the production of phthalic anhydride via the oxidation of naphthalene. It is shown that the requirements formulated can be used for the design of the reactor and for its immediate adjustment to a change in operating conditions. In view of the special behaviour of consecutive reactions in a tubular reactor a fine tuning of the operating conditions remains necessary after this adjustment.
\end{abstract}

\section{INTRODUCTION}

In a previous article [1] we discussed the safe design of cooled, tubular reactors for a set of exothermic, first order parallel reactions. We demonstrated that, with a set of dimensionless numbers characterizing the reaction system and which are independent of the operating and design parameters of the tubular reactor itself, a set of criteria could be derived, which related uniquely the values of operating and design parameters with the required selectivity of the reactor and with the reaction system parameters. The same method will now be applied to a set of exothermic, first order consecutive reactions; we refer to the previous article [1] for a discussion of the relevant dimensionless numbers as well as for a review of the literature and the basic philosophy of the method applied. The parameters describing the reaction system are $k_{R}, T_{R}, \gamma_{p}, p$ and $H$; the design and operating parameters are $\Delta \tau_{a d}$, $D a, \tau_{c}, \tau_{0}$ and $U^{*}$. These parameters are defined in the list of symbols.

Consecutive reactions differ from parallel reactions in various aspects. Firstly the selectivity is highest for the intermediate product $P$ in consecutive reactions at the onset of the reaction. This implicates that, if the reactant can be recovered from the reactor product and recycled to the reactor inlet, the reactor has to be operated at low conversions and high reactant recycle ratios in order to achieve a high sclectivity towards the desired product. The choice of the conversion and the corresponding recycle ratio is then only dominated by plant economics. This will not be discussed here.

However, in case the reactant cannot be recovered from the reactor product mixture, the reactor yield instead of the selectivity is dominating the plant performance. The yield is defined as the amount of desired product obtained per unit of key reactant fed to the reactor. Therefore, we will limit ourselves here to

\footnotetext{
tAuthor to whom correspondence should be addressed.
}

achieving a desired yield for a consecutive reaction system, for which the non-converted reactant cannot be recovered from the reactor product.

An important dimensionless parameter describing the reaction system is $p$, the ratio of the activation energy of the undesired to that of the desired reaction. In case $p<1$ the highest possible reactor temperatures should be chosen to achieve high yields. Here we will limit ourselves to consecutive reaction systems with $p>1$, where a low temperature level should be chosen in order to achieve high yields, despite the longer residence times required at the corresponding lower reaction rates.

Further we have to bear in mind that for consecutive reactions the maximum yield is reached at one definite value of the residence time or $D a_{\text {opt }}$, which fixes the reactor tube length $L$ with respect to the reactor load $u$. At this value of $D a_{\text {opt }}$ the differential selectivity has just reached the value of zero and the reaction has to be stopped. The value of $D a_{\text {opt }}$ depends on the shape of the temperature profile, or in other words on the history of the reaction mixture flowing through the reactor. For parallel reactions it was not necessary to stop the reaction after a certain residence time in order to achieve a maximum yield.

\section{DEVELOPMENT OF A CRITERION AND REOUIREMENTS}

\section{Basic equations}

We consider a tubular reactor in which two consecutive reactions occur:

$$
\boldsymbol{A} \rightarrow \boldsymbol{P} \rightarrow \boldsymbol{X} \text {. }
$$

In these reactions $A$ is the reactant, $P$ is the desired and $X$ is the undesired product. Both reactions are irreversible, exothermic and of the first order. The conversion rates are given by:

$$
R_{w A}=-k_{P} C_{A}
$$




$$
\begin{aligned}
& R_{w X}=k_{X} C_{P} \\
& R_{w P}=k_{P} C_{A}-k_{X} C_{P} .
\end{aligned}
$$

Here $\boldsymbol{R}_{w}$ is expressed in moles converted per unit time and per unit of mass of catalyst.

We will use the pseudo-homogeneous, onedimensional model of the cooled, tubular reactor, which is based on the following assumptions:

-the reactor is a plug flow reactor, in which the only transport mechanism is operating in the axial direction, hence there are no concentration gradients in the radial direction

- the temperature of the cooling medium $T_{c}$ is constant

-the physical and chemical data $\rho_{g}, \rho_{B}, c_{p}, \Delta H$ and $U$ are independent of temperature.

The mass and heat balances for this reactor model are:

$$
\begin{aligned}
u \frac{\mathrm{d} C_{A}}{\mathrm{~d} z}= & R_{w A} \rho_{B} \\
u \frac{\mathrm{d} C_{X}}{\mathrm{~d} z}= & R_{w X} \rho_{B} \\
u \frac{\mathrm{d} T}{\mathrm{~d} z}= & \left(-\Delta H_{A} R_{w A}+\Delta H_{X} R_{w X}\right) \frac{\rho_{B}}{\rho_{g} c_{p}} \\
& -\frac{4 U}{d_{\imath} \rho_{\theta} c_{p}}\left(T-T_{c}\right) .
\end{aligned}
$$

Since the definition of the reaction model is similar to the one introduced by Westerterp and Ptasinsky [1], eqs (5)-(7) can be made dimensionless and transformed the very same way, resulting in the following set of basic equations:

$$
\begin{aligned}
\frac{\mathrm{d} X_{A}}{\mathrm{~d} Z}= & \operatorname{Da\kappa }\left(1-X_{A}\right) \\
\frac{\mathrm{d} X_{P}}{\mathrm{~d} Z}= & \operatorname{Da}\left[\kappa\left(1-X_{A}\right)-\kappa^{p} X_{P}\right] \\
\frac{\mathrm{d} \tau}{\mathrm{d} Z}= & \operatorname{Da\Delta } \Delta \tau_{a d}\left[\kappa\left(1-X_{A}\right)+H \kappa^{p} X_{P}\right] \\
& -D a U *\left(\tau-\tau_{c}\right)
\end{aligned}
$$

together with the additional equation:

$$
\boldsymbol{X}_{\boldsymbol{A}}=\boldsymbol{X}_{P}+\boldsymbol{X}_{\boldsymbol{X}} \text {. }
$$

This set of equations describes the behaviour of consecutive reactions in our tubular reactor.

\section{Behaviour of the $\tau-X_{A}$ functions, the trajectories}

In order to investigate the temperature behaviour of the reactor, the system of the three eqs (8) (10) can be reduced to:

$$
\begin{aligned}
\frac{\mathrm{d} X_{P}}{\mathrm{~d} X_{A}}= & 1-\frac{\kappa^{p-1} X_{P}}{1-X_{A}} \\
\frac{\mathrm{d} \tau}{\mathrm{d} X_{A}}= & \Delta \tau_{a d}\left(1+H_{\kappa^{p-1}} \frac{X_{P}}{1-X_{A}}\right) \\
& -\frac{U *\left(\tau-\tau_{c}\right)}{\kappa\left(1-X_{A}\right)} .
\end{aligned}
$$

Unlike the system of two parallel reactions, the case of consccutive reactions cannot be reduced to one differential equation only, describing the $\tau-X_{A}$ behaviour. In fact, the reaction trajectories can only be obtained by simultaneous numerical integration of (12) and (13). Therefore, the $\tau-X_{A}$ trajectories have to be represented as curves in the three-dimensional space $\left(\tau, X_{A}, X_{P}\right)$ or $\left(\tau, X_{A}, X_{X}\right)$, whereas for parallel reactions the use of a two-dimensional plot was sufficient. This space is limited by: $\tau \geqslant \tau_{c}, 0 \leqslant X_{A} \leqslant 1$ and $0 \leqslant X_{X} \leqslant X_{A}$.

The characteristic maximum temperature in the hot spot of the reactor is defined by setting $d \tau / d X_{A}$ to zero in (13):

$$
\begin{aligned}
\left(X_{P}\right)_{m}+\left(X_{X}\right)_{m}=\left(X_{A}\right)_{m}= & 1+H \kappa_{m}^{p-1}\left(X_{P}\right)_{m} \\
& -\frac{U}{\Delta \tau_{a d}} \frac{\tau_{m}-\tau_{c}}{\kappa_{m}} .
\end{aligned}
$$

In the three-dimensional space, (14) is the equation of a surface, in which the maximum temperature $\tau_{m}$ depends on the value of the unknown parameter $\left(X_{P}\right)_{m}$, which, in turn, is influenced by the starting or inlet conditions of the reaction path and the value of $\tau_{c}$ and can only be found by simultaneous integration of (12) and (13). The necessity to use the three-dimensional space seriously hampered the understanding of the consecutive reaction system. Therefore, in order to reduce the complexity of the analysis of the temperature-conversion relationship and to find a solution similar to the one obtained for parallel reactions $[1,2]$, we looked for possible simplifications. With eq. (11) the locus plane eq. (14) can be rewritten as:

$$
\begin{array}{r}
\left(X_{A}\right)_{m}=\frac{1}{1-H \kappa_{m}^{p-1}}\left(1-\frac{U^{*}}{\Delta \tau_{a d}} \frac{\tau_{m}-\tau_{c}}{\kappa_{m}}\right) \\
-\frac{H}{\kappa_{m}^{1-p}-H} X_{X} .
\end{array}
$$

We found, after studying the trajectories, for all the possible practical values of the reaction system parameters that in the hot spot of the reactor almost no $X$ has yet been formed, provided that the reactor operates safely and that high yields towards desired product $\left(\theta_{p}>0.5\right)$ are obtained. This means that the second term in the right hand side of (14a) is negligible small. Only in the case of operating at runaway conditions or very close to runaway, considerable amounts of $X$ had been formed already in and before the hot spot. As our goal is to find criteria for high yields under stable operating conditions, it is therefore allowed to write for (14a):

$$
\left(X_{A}\right)_{m}=\frac{1}{1-H \kappa_{m}^{p-1}}\left(1-\frac{U^{*}}{\Delta \tau_{a d}} \frac{\tau_{m}-\tau_{c}}{\kappa_{m}}\right)
$$

which results in a two-dimensional approximate locus curve. Considering this expression, we have to realize that the heat production rate at any place in the reactor is equated to its maximum value because, if some $\boldsymbol{X}$ has been formed already, $X_{P}<X_{A}$ and consequently the local heat production rate $\Delta \tau_{a d}\left[\kappa\left(1-X_{A}\right)+H \kappa^{p} X_{P}\right]$ 
will be lower than the value used in (15). We also studied other possible simplifications, but best worked the assumption $X_{P}=X_{A}$, which is, due to a slight overestimation of the heat effect in the hot spot area, on the conservative side.

The locus of maxima curve, defined by (15), as well as some reaction trajectories are plotted in Fig. 1. It should be noticed that to the left side of the locus curve the derivative $\mathrm{d} \tau / \mathrm{d} X_{A}$ of a trajectory is always positive, while to the right we have decreasing temperatures at increasing conversion. At higher temperatures, especially as the term $H \kappa_{m}^{p-1} \rightarrow 1$, eq. (15) fails to give an accurate estimate for $X_{A}$ in the hot spot, for in that region the assumption $X_{P}=X_{A}$ is far too conservative. We should, of course, bear in mind that because of the high rates of the secondary reaction in that region and the corresponding low yields of the desired product, these high temperatures are out of the scope of our subject because in this case yields are generally lower than $50 \%$.

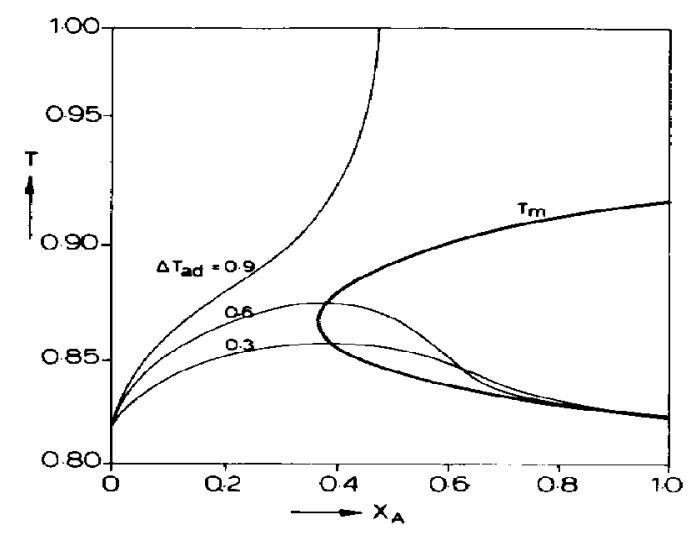

Fig. 1. Temperature trajectories in the reactor and locus of maxima curve; $p=2, H=2, \gamma_{p}=15, U^{*} / \Delta \tau_{\text {ad }}=1.5$.

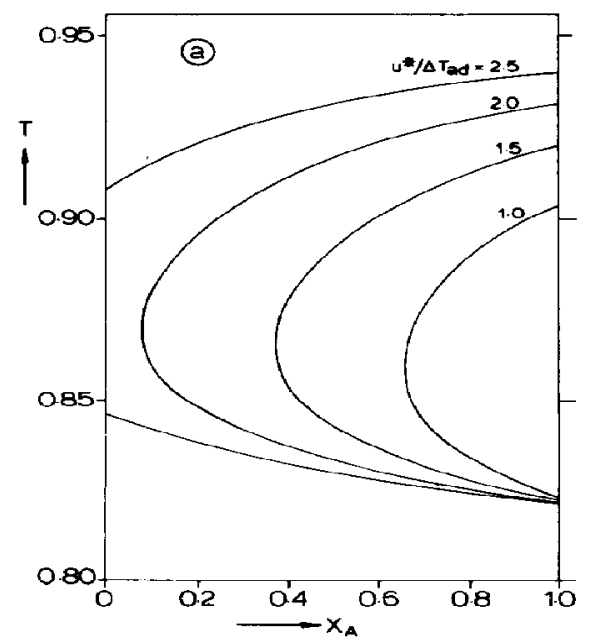

Analogous to the case of the parallel reactions, the shape and position of the curves is controlled by the reaction parameters $\gamma_{p}, p, H$ and the two design parameters $\tau_{c}$ and $U^{*} / \Delta \tau_{a d}$. Figure 2 shows that at increasing the ratio $U^{*} / \Delta \tau_{a d}$ or at decreasing $\tau_{c}$, the curves shift to the left, decreasing the dangerous region where $d \tau / d X_{A}>0$. At certain combinations of values, the curves start to intersect the $X_{A}=0$ axis, as was the case for parallel reactions.

\section{Yield criterion}

In order to develop a yield criterion, that permits us to select suitable operating and design parameters, we have to know the influence of the reactor temperature on the reactor performance. It is difficult to make general statements for real reactors with hot spots and temperature profiles, but the isothermal reactor can be considered as an idealized extreme, which can not be achieved in practice. Therefore, we consider the isothermal reactor, for which eqs (8)-(10) can be solved, and find for the maximum yield the relation:

$$
\left(\theta_{P}\right)_{\mathrm{opt}}=\left(\kappa^{p-1}\right)^{\frac{\kappa^{p-1}}{1-\kappa^{p-1}}}
$$

where $\kappa$ is taken at the reactor temperature of the isothermal reactor. The maximum yield of the desired product at isothermal conditions is a function of temperature only, for a specified set of values for $p$ and $\gamma_{p}$. At $\tau=1$ all combinations of $p$ and $\gamma_{p}$ lead to the same value of $\left(\theta_{p}\right)_{\mathrm{opt}}=1 / e=0.37$. For $p>1$ the maximum allowable temperature $\tau_{m a}$ is lower than unity. The conversion of reactant $A$, reached at the maximum value of the yield is given by:

$$
\left(X_{A}\right)_{\mathrm{opt}}=1-\left(\theta_{P}\right)_{\mathrm{opt}}^{\frac{1}{\kappa^{2-1}}} .
$$

At isothermal operating conditions the differential

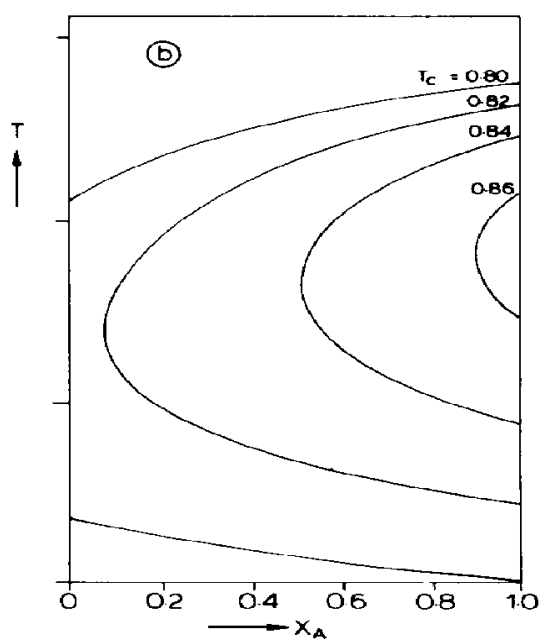

Fig. 2. Locus of maxima curves for a few values of the parameters: a: $U * / \Delta \tau_{a d}$ and $b: \tau_{c}$; further $p=2, H=2$, $\gamma_{p}=15$. 
selectivity $\mathrm{d} X_{P} / \mathrm{d} X_{A}$ is decreasing with increasing $X_{A}$; at the value $X_{A}=\left(X_{A}\right)_{u p t}$ the differential selectivity is just zero, while beyond this value there is a net consumption of $P$. The required residence time to reach the point of maximum yield at isothermal conditions is given by:

$$
D a_{\mathrm{opt}}=\frac{\ln \left(1-\left(X_{A}\right)_{\mathrm{opt}}\right)}{\kappa}=-\frac{\ln \left(\theta_{\mathrm{P}}\right)_{\mathrm{opt}}}{\kappa^{P}} .
$$

From eq. (16) we now can derive the reactor temperature $\tau_{m a}$ required to obtain a certain desired yield in an isothermal reactor, by solving equation (16) by trial and error, because we have the following relationship between $\kappa$ (from (16)) and $\tau_{m a}$ :

$$
\tau_{m a}=\left(1-\ln \kappa / \gamma_{p}\right)^{-1} \text {. }
$$

The derivation of this yield criterion is similar to the method previously developed for the selectivity criteria of parallel reactions [1]. The requirements proposed to meet the criterion depend upon the shape of the locus curve (15), which, because of its underlying conservative assumption for the rate of heat production, will always lead to a conservative value. The requirements are based on the assumption that reactor temperatures are nct allowed to surpass $\tau_{m a}$. If the requirements are established for the actual reactor, the $\tau_{m a}$ temperature is allowed to be reached in the hot spot only; as a consequence the actual yield will always be higher than the yield in an isothermal reactor operating at $\tau_{\text {ma }}$. Given the value $\tau_{\operatorname{ma}}$, two requirements for the reactor conditions are obtained that meet the yield criterion under different locus curve types.

The first requirement is now based, analogous to parallel reactions, on the locus curve intersecting the $X_{A}=0$ axis. It depends on the value of $\tau_{m a}$, which may not be surpassed in order to obtain a reactor yield equal to or greater than $\left(\theta_{p}\right)_{\text {opt }}$. The value of the intersection temperature is a result of a combination of values of $U^{*} / \Delta \tau_{a d}$ and $\left(\tau_{m a}-\tau_{c}\right)$, in such a way that $\tau_{m a}$ coincides with either the upper or the lower values of the temperature of intersection, as was shown in [1]. The reactor temperatures for any value of $\tau_{0} \leqslant \tau_{m a}$ will then always remain below $\tau_{m a}$. Expressed mathematically we have to introduce $\tau_{m a}$ in eq. (15) and equate $X_{A}$ to zero, resulting in the requirement.

$$
\frac{U^{*}}{\Delta \tau_{a d}}\left(\tau_{m a}-\tau_{c}\right) \geqslant \kappa_{m a} .
$$

The second requirement is derived from the locus curves (15) which do not intersect the $X_{A}=0$ axis. The requirement is based on the property of the trajectories that they start at the inlet conditions $\tau=\tau_{0}, X_{A}=0$ at their maximum slope and that beyond this point the slope decreases, due to the heat exchange with the cooling medium, provided runaway does not occur, which is the same as that the trajectory does not exhibit an inflexion point [3]. Hence, our requirement is based on the assumption:

$$
\left(\frac{\mathrm{d} \tau}{\mathrm{d} X_{A}}\right)_{X_{A}=0} \leqslant \frac{\tau_{m a}-\tau_{0}}{\left(X_{A}\right)_{m a}} .
$$

Substituting (21) in (13), equating $X_{P}=\left(X_{A}\right)_{\operatorname{ma}}$ and elimination of $\left(X_{A}\right)_{\operatorname{mu}}$ by means of $(15)$, we get for the second requirement:

$$
\Delta \tau_{a d} \leqslant \frac{\left(\tau_{m a}-\tau_{o}\right)\left(1-H \kappa_{m a}^{p-1}\right)}{1-\frac{U^{*}}{\Delta \tau_{a d}} \frac{\left(\tau_{m a}-\tau_{c}\right)}{\kappa_{m a}}}+\frac{U^{*}\left(\tau_{0}-\tau_{c}\right)}{\kappa_{0}} .
$$

In practice the inlet temperature $\tau_{o}$ often equals the coolant temperature $\tau_{c}$. For this case the second requirement becomes after rearrangement:

$$
\frac{U^{*}}{\Delta \tau_{a d}}\left(\tau_{m a}-\tau_{c}\right) \geqslant \kappa_{m a}\left[1-\left(1-H \kappa_{m a}^{p-1}\right) \frac{\tau_{m a}-\tau_{c}}{\Delta \tau_{a d}}\right] \text {. }
$$

It can be concluded from a comparison of the two requirements, that they differ in the term $[1-(1$ $\left.\left.-H \kappa_{m a}^{p-1}\right)\left(\tau_{m a}-\tau_{c}\right) / \Delta \tau_{a d}\right]$ which term is always $<1$ for high yields.

Therefore the first requirement can be considered as a more stringent version of the second one. Analogous to parallel reactions we can see that the product of the dimensionless parameters $U^{*} / \Delta \tau_{a d}$ and $\left(\tau_{m a}-\tau_{c}\right)$ has to be kept higher than a fixed minimum value defined by one of the requirements, in order to achieve the desired or a better yield. We have to realize the importance of the coolant temperature $\tau_{c}$, for low values of $\tau_{c}$ will result in large reactor lengths because of the low average temperature level in the reactor.

Selection of practical values for the cooling medium temperature

Together with a maximum allowable temperature we have to specify the optimum value of $D a$, the residence time in the reactor. As already stated previously the selectivity $\mathrm{d} X_{P} / \mathrm{d} X_{A}$ of the reactor decreases with increasing conversion. The optimum reactor design should now result in reactor outlet conditions that coincide with the point of maximum yield. Because the maximum reactor yield depends on the temperature profile in the reactor, the point where $\mathrm{d} X_{P} / \mathrm{d} X_{A}=0$ can only be obtained by numerical integration. An approximation of the value of $D a_{\text {opt }}$ can be obtained from the $\left(\theta_{P}\right)_{\text {opt }}$ value for an isothermal reactor, defined by (18). However, by varying the temperature we also vary the values of $\left(\theta_{P}\right)_{\text {opt }}$. This gives us two different ways for the selection of a range of practical cooling medium temperatures.

The first is the ratio of the optimum Damköhler numbers, taken at the maximum allowable temperature and at the coolant temperature. This ratio is then a measure of the increase in reactor length due to both the lower reactor temperature level and the higher maximum yield achieved at the lower temperature. A somewhat simpler way to compare the increase in reactor length is to consider the isothermal reactor at $\tau_{c}$, at the same conversion $X_{A}$ as at $\tau_{m a}$, defined by (18). Substitution of $\tau_{c}$ and $\tau_{m a}$ and division results in a ratio for the reactor residence times in isothermal reactors of

$$
\frac{\left(D a_{\mathrm{opt}}\right)_{c}}{\left(D a_{\mathrm{opt}}\right)_{m a}}=\frac{D a_{c}}{D a_{m a}}=\left(\frac{\kappa_{m a}}{\kappa_{c}}\right) \text { for constant } X_{A} .
$$


The actual ratio of residence times, for real reactors with a temperature profile will now be somewhere in between. The ratio $D a_{c} / D a_{m a}$ as a function of $\left(\tau_{m a}-\tau_{c}\right)$ and of varying values of parameters $\gamma_{p}, p$ and $\left(\theta_{P}\right)_{\text {opt }}$ was studied over the entire range of practical values of the parameters. It was observed that above a certain value of $\left(\tau_{\operatorname{ma}}-\tau_{c}\right)$ the ratio becomes very sensitive to variations in the coolant temperature. The boundary of this sensitivity is in the range of $D a_{c} / D a_{m a}$ from 3-5. Therefore we will use for the minimum allowable isothermal reactor temperature $\left(\tau_{\mathrm{c}}\right)_{\min }$ a value at which the ratio $D a_{c} / D a_{m a}$ equals 5 for cheap or 3 for expensive reactors. For a fixed value of $D a_{c} / D a_{m a}$ and a fixed maximum allowable temperature $\tau_{m a}$ calculated by trial and error from (16) and (19), the corresponding value of $\tau_{c}$ can be calculated by rearranging (18) with (24) to:

$$
\begin{aligned}
\left(\tau_{c}\right)_{\min }=\frac{\gamma_{p}}{\ln \left(D a_{c} / D a_{m a}\right)+\gamma_{p} / \tau_{m a}} \\
\text { with } \quad D a_{c} / D a_{m a} \simeq 3-5 .
\end{aligned}
$$

The influence of the dimensionless number $U^{*} / \Delta \tau_{\text {ad }}$

Intuitively, it can be felt that the local heat production rate will reach a maximum value near the hot spot in the reactor. This was confirmed by calculations. If $\tau_{m}$ is the hot spot temperature, we can also feel intuitively that for small values of $\tau_{m}-\tau_{c}$, the required value of $U^{*}$ must be large in order to keep $\tau_{m}-\tau_{c}$ at the required low value, despite the low reaction rates. On the other hand, for large values of $\tau_{m}-\tau_{c}$, where $\tau_{m}$ and the local heat production rates near the hot spot are high, the required value of $U^{*}$ should also be high. This leads to the conclusion that somewhere in between must be a minimum value of $U^{*}$ to maintain $\tau_{m}$ at the required value. This minimum value can be found by calculating the reactor temperature rise from the reactor inlet (at $\tau_{o}=\tau_{c}$ ) to the hot spot, while no heat is removed at all; in this case the adiabatic temperaturc rise is $\tau_{m}-\tau_{c}=\Delta \tau_{a d}\left(X_{A}+H X_{X}\right)$. As stated before, for stable operation outside the runaway region $X_{X}$ before the hot spot is practically zero, so that for $X_{X}=0$ we can define the conversion in the hot spot by:

$$
X_{A}=\frac{\tau_{m}-\tau_{c}}{\Delta \tau_{a d}}
$$

Substitution of (26) into (15) gives:

$$
\frac{U^{*}}{\Delta \tau_{a d}}=\frac{\kappa_{m}}{\tau_{m}-\tau_{c}}+\frac{H \kappa_{m}^{p}-\kappa_{m}}{\Delta \tau_{\alpha d}}
$$

This expression (27) will lead to too conservative values for $U^{*}$, because of the underlying assumption of no heat removal before the hot spot, which leads to too high values of $X_{A}$ in the hot spot. On the other hand, as calculations have shown the hot spot to be situated near the reactor inlet, it will also lead to realistic values for $U^{*}$.

The desired minimum value of $U^{*} / \Delta \tau_{\text {ad }}$ can be found by equating the derivative $\partial\left(U^{*} / \Delta \tau_{a d}\right) / \partial \tau_{m}$ in
(27) to zero:

$$
\begin{aligned}
\frac{\partial\left(\frac{U^{*}}{\Delta \tau_{a d}}\right)}{\partial \tau_{m}}= & \left(\frac{\gamma_{p} \kappa_{m}}{\tau_{m}^{2}}\right)\left(\frac{H p \kappa_{m}^{p-1}-1}{\Delta \tau_{a d}}\left(\tau_{m}-\tau_{c}\right)^{2}\right. \\
& \left.+\left(\tau_{m}-\tau_{c}\right)-\frac{\tau_{m}}{\gamma_{p}}\right)=0
\end{aligned}
$$

which leads to the formulation of the third requirement:

$$
\left(\frac{H p \kappa_{m}^{p-1}-1}{\Delta \tau_{a d}}\right)\left(\tau_{m}-\tau_{c}\right)^{2}+\left(\tau_{m}-\tau_{c}\right)-\frac{\tau_{m}^{2}}{\gamma_{p}}=0
$$

Once $\tau_{c}$ has been set between $\tau_{m a}$ and $\left(\tau_{c}\right)_{\min }, \tau_{m}$ can be calculated by trial and error from (29). Substitution of this value of $\tau_{m}$ into (27) gives the minimum value of $U^{*}$, corresponding to the chosen value of $\tau_{c}$, and a requirement for optimum tube diameters. Vice versa, for a given value of $\tau_{m a}=\tau_{m}$ the corresponding values of $\tau_{c}$ and $U^{*}$ can be calculated by trial and error. How the three requirements work out is shown in Fig. 3, in which the runaway conditions were found by numerical integration. The advantage of the third requirement, as can be concluded from the figure, is it being the least stringent, while on the other hand it is only based on the coolant temperature and reaction system parameters and the reactant concentration $\left(\Delta \tau_{a d}\right)$. Furthermore it will always lead to safe reactor operating conditions because of the underlying conservative assumptions.

\section{The relation between the integral yield and isothermal reactor yields}

How the requirements equations (20) and (23) work out for a given set of parameter values has been studied by numerical integration of eqs (12) and (13). We calculated the reactor yields for a wide range of reaction parameters $p, \gamma_{p}, H$ and operating variables $D a_{c} / D a_{m a}, \Delta \tau_{a d}$ and $\left(\theta_{P}\right)_{\text {opt }}$. In all our calculations we assumed the inlet condition $\tau_{0}=\tau_{c}$. The results for both requirements are practically equal; Fig. 4 shows the typical relation between $\theta_{P}$ and $\left(\theta_{P}\right)_{\text {opt }}$ as a function of $D a_{c} / D a_{m a}$ and $p$. A discussion of the influence of all

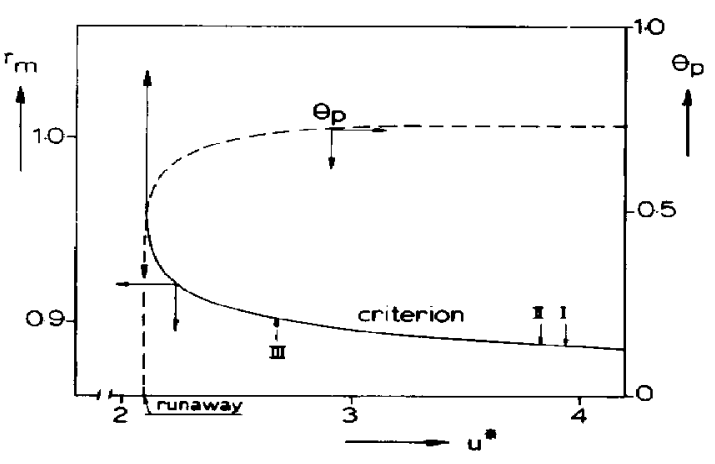

Fig. 3. Influence of $U^{*}$ on the hot spot temperature and on the yield; $p=2, H=2, \gamma_{p}=15$ and $\Delta \tau_{\mu}=0.5$. The points $I$, II and III correspond to the values of $U^{*}$ as calculated for the first, second and third requirements to meet the criterion. 


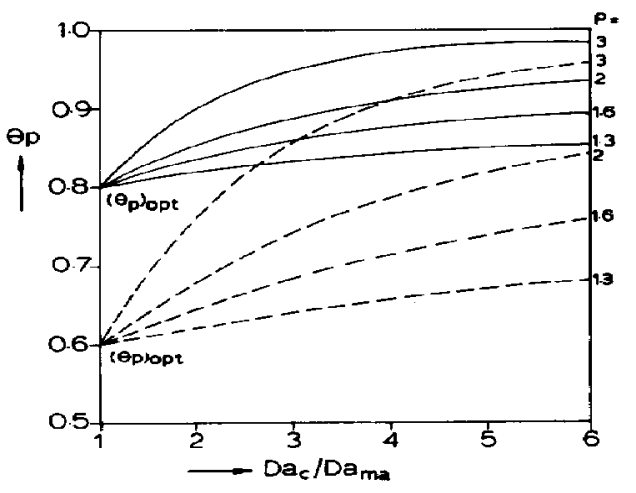

Fig. 4. Comparison of the computed integral yield and the isothermal yield for varying values of $D a_{c} / D a_{m a}$ and $p$, if the first requirement is used. Further $p=2, H=2, \gamma_{p}=15$ and $\Delta \tau_{a d}=0.5$. The isothermal yields are 0.6 and 0.8 respectively.

the parameters is given later on; for the requirements, however, only a variation of $D a_{c} / D a_{m a}$ and $p$ have a significant influence.

Check for parametric sensitivity

We still have to check whether the three requirements do not lead us into the region of high parametric sensitivity, resulting in runaway of the reactor temperatures. Analogous to parallel reactions, the first requirement is inherently insensitive, sensu strictu this reactor cannot be unstable [1]. As for the second and third requirement the situation is more complicated and has to be analysed in more detail. This analysis of the behaviour of the second derivative of the $\tau-X_{A}$ function is very similar to that for parallel reactions [2]. We investigated the temperature behaviour in the ranges: $1.2<p<4.0 ; 5<\gamma_{p}<80$; $0.5<H$ $<5.0 ; 0.01<\Delta \tau_{a d}<1.5 ; 1.5<D a_{c} / D_{m a}<8$ and 0.5 $<\left(\theta_{P}\right)_{\text {opt }}<0.95$. Within this wide range of variation of each parameter, we found that runaway does not occur if the second or third requirement is adhered to. Therefore we can conclude that the trajectories calculated according to the requirements in this article fall in the region of low parametric sensitivity.

\section{Discussion of the influence of the relevant dimensionless numbers}

From the three requirements developed (eqs 20, 23 and 29) we can conclude that the dimensionless numbers $\tau_{m a}-\tau_{c}, p, \gamma_{p}, H, \Delta \tau_{a d}$ and $U^{*}$ govern the behaviour of the cooled tubular reactor. The third requirement, after a coolant temperature $\tau_{c}$ had been chosen, led to an optimal choice of $U^{*} / \Delta \tau_{\text {ad }}$ which reaches a minimum value. At this minimum value the maximum allowable tube diameter is found. We will use this third requirement to evaluate the influence of the relevant dimensionless numbers.

The choice of $D a_{c} / D a_{m a}$. A correct choice of $D a_{r} / D a_{m a}$ can only be made on economic grounds: the investment and operating costs of the reactor. Once $\tau_{c}$ has been chosen, with requirement three the minimum value of $U^{*} / \Delta \tau_{a d}$ can be determined together with the corresponding value of $\tau_{m}$. In Fig. 5(a) and in Table 1
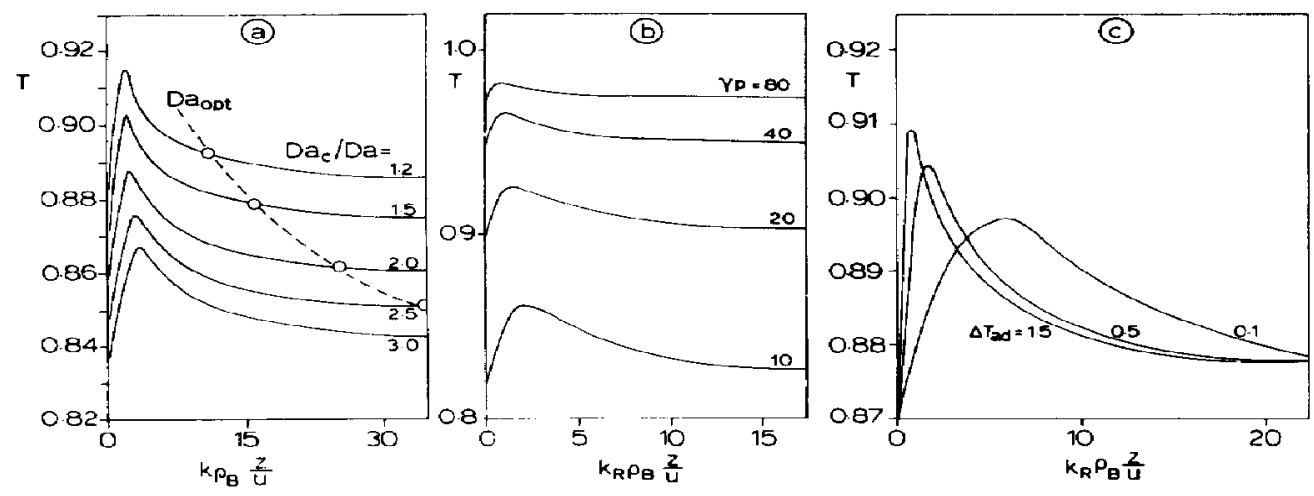

Fig. 5. Influence of the dimensionless numbers $D a_{c} / D a_{m a}$ (a) $\gamma_{p}$ (b) and $\Delta \tau_{a d}$ (c) on the temperature profiles in the reactor using requirement 3 . Further $p=2, H=2, D a_{c} / D a_{m a}=1.5(\mathrm{~b}, \mathrm{c}), \gamma_{p}=15(\mathrm{a}, \mathrm{c}), \Delta \tau_{\text {ad }}=0.5$ (a, b).

Table 1. Influence of the ratio $D a_{c} / D a_{m a}$ on the reactor behaviour

\begin{tabular}{ccccccc}
\hline \multicolumn{7}{c}{$\left(\theta_{p}\right)_{\text {opt }}=0.7, \tau_{m a}=0.983, p=2, H=2, \gamma_{p}=15, \Delta \tau_{\text {ad }}=0.5$} \\
\hline$D a_{c} / D a_{m a}$ & $\tau_{c}$ & $\tau_{m}$ & $U^{*}$ & $D a_{\text {opt }}$ & $X_{A, \text { opt }}$ & $X_{\text {P,opt }}$ \\
\hline 1.5 & 0.872 & 0.928 & 2.67 & 16 & 0.907 & 0.722 \\
2.0 & 0.858 & 0.913 & 2.04 & 25 & 0.931 & 0.769 \\
2.5 & 0.847 & 0.902 & 1.66 & 34 & 0.942 & 0.801 \\
3.0 & 0.838 & 0.893 & 1.40 & 44 & 0.951 & 0.815 \\
\hline
\end{tabular}

Only for the third requirement (29) the yield at very low values of $\mathrm{Da} / \mathrm{Da}$ ma can become somewhat lower than the desired one. 
for a certain set of values of the dimensionless groups and for an isothermal value of $\left(\theta_{P}\right)_{\text {opt }}=0.70$ the results are shown. With increasing values of $D a_{c} / D a_{m a}$ the tube lengths and their diameters get larger and also the yield obtained increases. For stable, safe operation the value of $\tau_{m a}-\tau_{c}$ is slowly reduced for increasing values of the ratio.

The influence of $\gamma_{p}$. The value of $\gamma_{p}=E_{P} / R T_{R}$ is a measure of the temperature influence on the reaction rates. The higher the value of $\gamma_{p}$ the more sensitive the reactions are for the reaction temperature; for high $\gamma_{p}$ values already near $T_{R}$ the reactions are slowed down sufficiently for safe operation. For low values of $\gamma_{p}$ the temperature sensitivity is low so that higher values of $\tau_{m}-\tau_{c}$ can be accepted for safe operation; this is shown in Fig. 5(b). So for increasing values of $\gamma_{p}$ and for one desired value of $\left(\theta_{P}\right)_{\text {opt }}$ :

- the required value of $\tau_{c}$ and the temperature level in the reactor increases

the allowable values of $\tau_{m}-\tau_{c}$ decrease and the hot spot becomes less distinct

- the required tube diameter decreases

- the required tube length is hardly affected.

The influence of $H$. The influence of $H=\Delta H_{A} / \Delta H_{X}$ is negligibly small. The same values for $\tau_{m}$ and for $\tau_{c}$ are required for a certain yield; for high values of $\boldsymbol{H}$ - high heat effect of the second reaction-the temperature level in the tail after the hot spot will be somewhat higher.

The influence of $p$. At decreasing the reactor temperatures for lower values of $p=E_{X} / E_{P}$ the reaction rate of the undesired reaction is less strongly reduced with respect to the desired one. Vice versa, for increasing values of $p$ :

- the required coolant temperature becomes higher

- the tube length becomes shorter and diameter smaller

-the allowable temperature difference $\tau_{m}-\tau_{r}$ gets more distinct.

The influence of $\Delta \tau_{\text {ad }}$. The influences of $\Delta \tau_{\text {ad }}$ $=\Delta H_{A} C_{A 0} / \rho_{\theta} c_{p} T_{R}$ or the concentration of the reactant in the feed hardly affects the tube length, $\tau_{c}$ and the integral yield obtained, the required tube diameter decreases proportionally with increasing $\Delta \tau_{a d}$ and the hot spot $\left(\tau_{m}-\tau_{c}\right)$ gets more distinct, see Fig. 5(c).

Procedure for the safe design of cooled tubular reactors for consecutive first order reactions

From the previous discussions now the following procedure for the safe design of cooled tubular reactors in which exothermic, consecutive first order reactions are executed, can be developed:

(1) For the reaction system considered determine $k_{R}, T_{R}$ and the other reaction system parameters $\gamma_{p}, H$ and $p$.

(2) Choose a required value of the integral yield.

(3) Determine $\tau_{m a}$ with eq. (16).
(4) Calculate different values of $T_{c}$ for different values of the ratio $D a_{c} / D a_{m a}$ with requirement (20) or (23). Further $T_{m a}>T_{c} \geqslant\left(T_{c}\right)_{\min }$ according to (25).

(5) Calculate with the third requirement (equation (29)) the minimum value of $U^{*} / \Delta \tau_{a d}$.

(6) For given feed concentrations $\Delta \tau_{a d}$, calculate values of $U$ and $d_{t}$ from $U^{*}$ at the chosen reactor load.

(7) Calculate numerically the required tube length and determine the tube lengths and tube diameters as a function of $\tau_{c}$.

(8) Repeat steps $2-8$ for different yields and reactant concentrations and determine the economically optimum reactor configuration.

(9) Also for a given value of $d_{t}$ and $U$, as determined by the reactor load, $U^{*}$ can be calculated. Moreover, if $T_{m a}$ and $\Delta T_{a d}$ are known, the value of $T_{c}$ satisfying requirement (20) or (23) can be calculated using a search routine.

We should warn that the method has been developed for first order reactions; for reactions of different order. and especially for reactions with Langmuir-Hinshelwood or Eley-Rideal kinetics the method will not work.

\section{THE DESIGN AND OPERATION OF A NAPHTHALENE OXIDATION REACTOR}

\section{Basic data}

Here we will apply the criterion to the oxidation of naphthalene. The industrial air oxidation of naphthalene over a $\mathrm{V}_{2} \mathrm{O}_{5}$ catalyst with an excess of oxygen produces the desired intermediate phthalic anhydride, which itself is susceptible to further oxidation towards the undesired $\mathrm{CO}_{2}$ and $\mathrm{H}_{2} \mathrm{O}$.

DeMaria, Longfield and Butler [4] presented kinetic data for two catalysts $A$ and $B$ and for a reaction network of the following character:

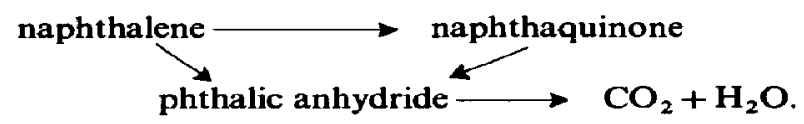

From their data Westerterp [5] and also later Carberry and White [6] derived a further simplified scheme of two consecutive reactions:

$$
\text { naphthalene } \stackrel{k_{2}}{\rightarrow} \text { phthalic anhydride } \stackrel{k_{x}}{\rightarrow} \mathrm{CO}_{2}+\mathrm{H}_{2} \mathrm{O} \text {. }
$$

According to [4] all reactions can be considered to be pseudo first order in naphthalene and phthalic anhydride respectively and zero order in oxygen, which is always in vast stoichiometric excess. Of the two catalysts tested in [4] catalyst $B$ with $p=E_{X} / E_{P}$ $=2.19$ is of interest to this study. The kinetic data are taken from [5] and are given in Table 2 together with the reaction system parameters and the chosen basic reactor design data as well.

\section{Determination of the tube diameter for a naphthalene} oxidation reactor

We will now use the requirements derived to determine safe tube diameters for a minimum yield of $\theta_{p}=0.7$ of phthalic anhydride. The reactor inlet 
temperature is assumed to be equal to $\tau_{c}$. The maximum allowable temperature $\tau_{m a}$ is calculated according to:

$$
\tau_{m a}=\frac{\gamma_{p}(p-1)}{\gamma_{p}(p-1)-\frac{1-\kappa_{\operatorname{ma}}^{p-1}}{\kappa_{m a}^{p}} \ln \theta_{p}}=0.90
$$

so that $T_{m a}=0.90 \times 770=693 \mathrm{~K}$. As was outlined before the actual yields obtained for this value of $T_{\text {mo }}$ will be higher than 0.7 , because the average temperature level in the reactor will be lower than $T_{\text {ma }}$. The minimum coolant temperature $\left(\tau_{c}\right)_{\min }$ can be calculated from:

$$
\tau_{c \min }=\frac{\gamma_{p}}{\ln \left(D a_{c} / D a_{\min }\right)+\gamma_{p} / \tau_{\operatorname{ma}}}
$$

resulting for $D a_{c} / D a_{\min }=5$ in $T_{c \min }=625 \mathrm{~K}$. The derived requirements can be rewritten as:

$$
\frac{U^{*}}{\Delta \tau_{a d}}\left(\tau_{m a}-\tau_{c}\right)>\kappa_{m a} P_{1,2,3}
$$

with $P_{1}=1.0$ for the first requirement and with $P_{2}$ and $P_{3}$ depending on the reactor conditions for the second and third requirement respectively. The reactor will be cooled with a molten salt mixture for which the heat transfer coefficient was determined to be $\alpha_{c}$
$=1500 \mathrm{~W} / \mathrm{m}^{2} \mathrm{~K}$ in the temperature range considered [7]. This value of $\alpha_{c}$ will further be kept constant in our considerations. The inside heat transfer coefficient as a function of a.o. the tube diameter, the temperature and the gas velocity has been calculated as outlined in [8], the overall heat transfer coefficients $U$ are given in Table 3.

For standard tube diameters and the basic design data of Table 2 we calculated the coolant temperature $T_{c}$ satisfying the requirements; results are reported in Table 3. We see from this table that for the basic design data and for $T_{c}>T_{c \text { min }}=625 \mathrm{~K}$ the largest allowable tube diameter is 1 in. and also that the second and third requirement are less stringent allowing higher coolant temperatures than first one.

With the values obtained for $T_{c}$ we can integrate the simultaneous linear differential equations to find the optimum reactor length, at which the phthalic anhydride yield reaches its maximum. The results are also given in Table 3. Further Fig. 6 presents the reactant conversion $X_{A}$, the reactor temperature $T$ and the yield $X_{P}$ as a function of the location in the reactor.

From the data obtained we now select a reactor tube of 1 in. dia. and of $4.0 \mathrm{~m}$ length. This corresponds to industrial practice [9]. For the basic design data and for the third requirement we then have $T_{c}=643 \mathrm{~K}$ and

Table 2. Values of system and design parameters for the naphthalene oxidation reactor

\begin{tabular}{|c|c|c|c|c|c|c|c|c|}
\hline \multicolumn{4}{|c|}{$\begin{array}{l}C_{\mathrm{AO}}=0.8 \text { mole } \% \\
\theta_{P}=0.7\end{array}$} & \multicolumn{3}{|c|}{$\begin{array}{l}u=1.30 \mathrm{~m} / \mathrm{s} \\
T_{m a}=693 \mathrm{~K}\end{array}$} & \multirow[b]{2}{*}{$X_{P, \text { opt }}$} & \multirow[b]{2}{*}{$\boldsymbol{X}_{\boldsymbol{A}, \mathrm{OP}}$} \\
\hline $\begin{array}{c}d_{t} \\
\text { (inch) }\end{array}$ & $\left(10^{\frac{d_{t}}{3}} \mathrm{~m}\right)$ & $\boldsymbol{P}$ & $\begin{array}{c}U \\
\left(W / m^{2} K\right)\end{array}$ & $\begin{array}{c}T_{c} \\
(\mathbf{K})\end{array}$ & $D a_{c} / D a$ & $\begin{array}{l}L_{\text {opl }} \\
(\mathrm{m})\end{array}$ & & \\
\hline \multicolumn{9}{|c|}{ First requirement } \\
\hline$\frac{1}{\frac{1}{3}}$ & 12.2 & 1.0 & 189 & 667 & 1.78 & 1.8 & 0.78 & 0.93 \\
\hline$\frac{3}{4}$ & 17.9 & 1.0 & 186 & 654 & 2.41 & 2.7 & 0.82 & 0.95 \\
\hline 1 & 24.3 & 1.0 & 182 & 639 & 3.49 & 4.6 & 0.87 & 0.96 \\
\hline \multicolumn{9}{|c|}{ Second requirement } \\
\hline$\frac{1}{2}$ & 12.2 & 0.97 & 189 & 668 & 1.75 & 1.8 & 0.77 & 0.94 \\
\hline$\frac{3}{4}$ & 17.9 & 0.96 & 186 & 656 & 2.29 & 2.4 & 0.81 & 0.95 \\
\hline $\mathbf{i}$ & 24.3 & 0.93 & 182 & 643 & 3.17 & 3.9 & 0.85 & 0.96 \\
\hline \multicolumn{9}{|c|}{ Third requirement } \\
\hline$\frac{1}{2}$ & 12.2 & 0.62 & 190 & 677 & 1.42 & 1.2 & 0.72 & 0.92 \\
\hline$\frac{3}{4}$ & 17.9 & 0.90 & 186 & 658 & 2.19 & 2.4 & 0.80 & 0.95 \\
\hline$i$ & 24.3 & 0.93 & 182 & 643 & 3.17 & 3.9 & 0.85 & 0.96 \\
\hline
\end{tabular}

$$
\begin{aligned}
& \text { Kinetic rate coefficients (kmoles } / \mathrm{m}^{3} \text { reactor volume s) } \\
& k_{P} \rho_{B}=6.46 \times 10^{6} \exp \left(-\frac{10100}{T}\right) k_{X} \rho_{B}=37.9 \times 10^{12} \exp \left(-\frac{22100}{T}\right) \\
& \text { Reaction system parameters } \\
& -\Delta H_{P}=1881 \mathrm{MJ} / \mathrm{kmole} \\
& k_{R} \rho_{B}=13 \mathrm{~s}^{-1} \\
& p=2.19 \\
& H=1.75 \\
& -\Delta H_{X}=3282 \mathrm{MJ} / \mathbf{k m o l e} \\
& T_{R}=770 \mathrm{~K} \\
& \gamma_{p}=13.5 \\
& c_{p}=1040 \mathrm{~J} / \mathrm{kg} \mathrm{K} \\
& \text { Basic design data } \\
& p=0.2 \mathrm{MPa} \\
& C_{A 0}=0.8 \text { mole } \% \\
& \rho_{g}=1.30 \mathrm{~kg} / \mathrm{m}^{3} \text { reaction mixture } \\
& u_{0}=1.30 \mathrm{~m} / \mathrm{s} \\
& \begin{array}{l}
\rho_{g}=1.30 \mathrm{~kg} / \mathrm{m}^{3} \\
d_{p}=4 \times 10^{-3} \mathrm{~m}
\end{array}
\end{aligned}
$$

Table 3. Reactor configurations designed according to the yield criteria 

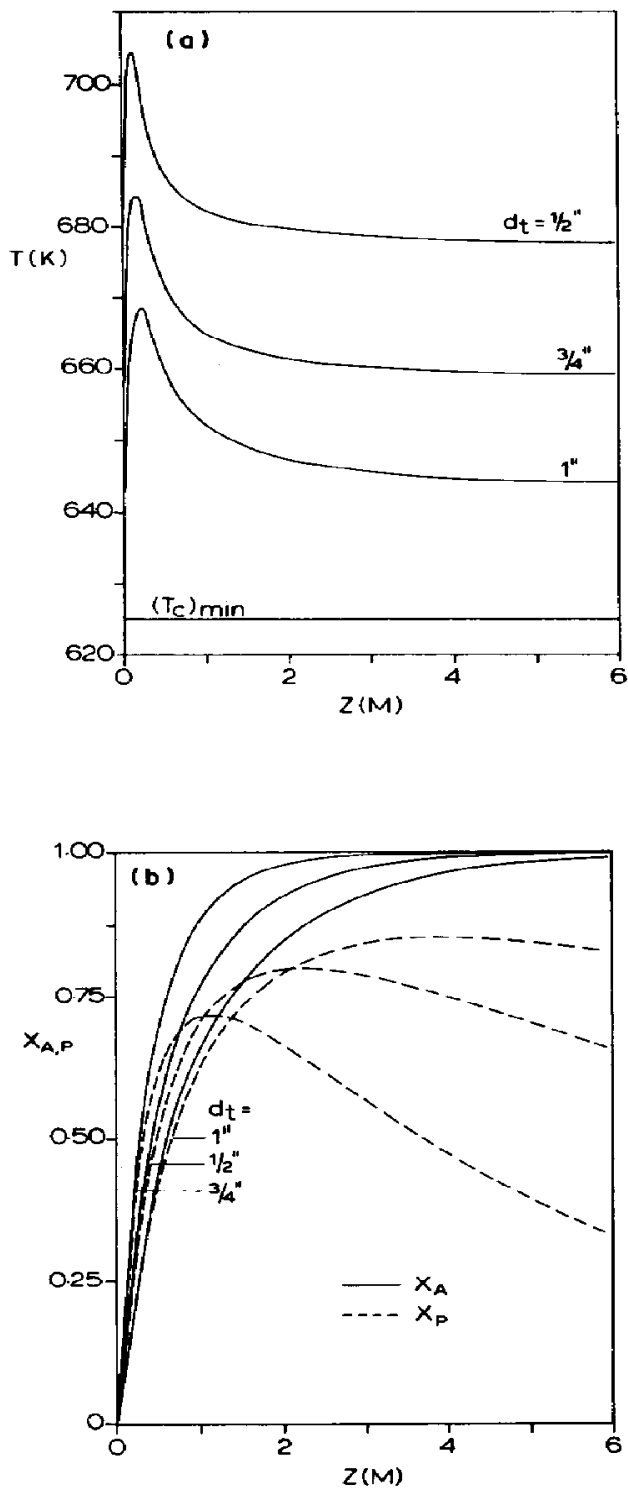

Fig. 6. Temperature (a) and conversion (b) profiles in reactors designed according to the third requirement, see Table 3 for further data.

$P_{3}=0.93$. After the required substitution we find for the reactor, that

$$
\frac{U\left(T_{m a}-T_{c}\right)}{d_{t} C_{A 0}}=1.25 \times 10^{9} \mathrm{~J} / \mathrm{kmole} \mathrm{s}
$$

should be kept constant during the operation of the reactor.

\section{Operation of the reactor}

Once a reactor has been designed both the diameter and the length of the tube are fixed. In the key dimensionless group $U^{*}\left(\tau_{m a}-\tau_{e}\right) / \Delta T_{a d}$ the reactant concentration $C_{A O}$ and the total heat transfer coefficient $U$ can deviate during operation considerably from the design conditions chosen. These changes then have to be compensated for with the operating variable $T_{c}$. This caused no problems for parallel reactions as discussed in $[1,2]$. For consecutive reactions on the contrary operational difficulties can be expected, because, e.g. for a different reactor load the maximum yield is reached at a tube length which will deviate from the actual length. After the reactor has been installed the optimum residence time $L / u$ can only be met at the design load. Fortunately the yield vs residence time curve is rather flat near the optimum. We will discuss the influence of changes in $C_{A 0}$ and $u$ and will demonstrate that an immediate adjustment to changes via the requirement $U\left(T_{m a}-T_{c}\right) / d_{t} C_{A 0}$ being constant, is possible after which a finer tuning with $T_{c}$ during operation is needed to reach a maximum yield again.

Inlet concentration changes and runaway. By increasing the reactant concentration in the feed and by keeping $T_{c}$ constant - that is not using the criterion we can check how far we are away from runaway. Calculations establish that runaway starts at $C_{A 0}$ $=1.05$ mole $\%$, this is $30 \%$ higher than design conditions.

If we do adhere to the criterion the yield is reasonably well maintained through adjustment of $T_{c}$, as shown in Table 4. So the criterion gives a good guideline for varying inlet concentrations.

Varying reactor loads. Market demand makes the reactor load vary continuously during plant life. This has an important impact on the reactor performance and we therefore, as an example, will consider two reactor load changes to 50 and $150 \%$ respectively of the design rate of $u=1.30 \mathrm{~m} / \mathrm{s}$. As a result the residence time in the reactor is affected and also the overall heat transfer coefficient changes considerably. The results are given in Table 5 .

First consider what happens if $T_{c}$ is not changed. For the lower load the hot spot temperature rises sharply and the reactor is close to runaway, which would be reached at $u=0.55 \mathrm{~m} / \mathrm{s}$, and also the yield drops sharply. At the higher load we observe also a yield decrease.

We now adjust $T_{c}$ according to our criterion. The results in Table 5 show that the negative effects of the load changes are largely compensated for. For the

Table 4. Required operating conditions at changing inlet concentrations

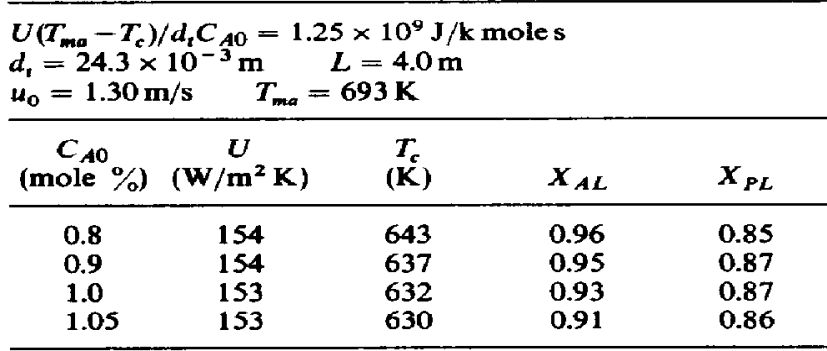


Table 5. Reactor performance at varying loads

\begin{tabular}{|c|c|c|c|c|c|}
\hline \multicolumn{2}{|c|}{$C_{\mathrm{A0}}=0.8$ mole $\%$} & \multicolumn{4}{|c|}{$L=4.0 \mathrm{~m} \quad d_{t}=24.3 \times 10^{-3} \mathrm{~m}$} \\
\hline$\stackrel{u}{u / s)}$ & $\begin{array}{c}U \\
\left(W / m^{2} K\right)\end{array}$ & $\begin{array}{c}\boldsymbol{T}_{\boldsymbol{c}} \\
\mathbf{( \mathbf { K } )}\end{array}$ & $\underset{(\mathbf{K})}{T_{m}}$ & $X_{A L}$ & $\boldsymbol{X}_{P L}$ \\
\hline \multicolumn{6}{|c|}{ Design conditions } \\
\hline 1.30 & 154 & 643 & 668 & 0.96 & 0.85 \\
\hline \multicolumn{6}{|c|}{ Load change and $T_{c}$ kept constant } \\
\hline 0.65 & 118 & 643 & 689 & 0.99 & 0.75 \\
\hline 1.95 & 186 & 643 & 662 & 0.89 & 0.80 \\
\hline \multicolumn{6}{|c|}{ Load change and criterion used } \\
\hline 0.65 & 116 & 631 & 655 & 0.99 & 0.88 \\
\hline 1.95 & 187 & 652 & 678 & 0.94 & 0.82 \\
\hline \multicolumn{6}{|c|}{ Cooling temperature tuned to maximum yield } \\
\hline 0.65 & 115 & 620 & 636 & 0.97 & 0.91 \\
\hline 1.95 & 186 & 646 & 667 & 0.91 & 0.83 \\
\hline
\end{tabular}

lower load we have to decrease $T_{c}-$ in [2] it has been discussed why this is so-and even observe a yield increase. For the higher load we have to increase $T_{c}$. However, we have to realise that the optimum reactor length for maximum yield no more coincides with the actual length. This requires a further adjustment of $T_{c}$, which in both cases has to be decreased. The final results of this fine tuning are also given in Table 5. From these results it becomes clear that the use of the criterion gives an excellent means to prevent runaway, and also provides a good starting point for further tuning by slowly reducing $T_{c}$ till the maximum yield is reached again.

\section{REMARKS}

We have shown that the criterion gives us good guidelines for the design and operation of a safe tubular reactor with consecutive reactions in order to achieve high yields and to prevent runaway. This presupposed that all variables are known exactly. Regretfully this is not true in industrial practice. Some remarks have to be made on the major uncertainties in handling the criterion in practice.

Heat transfer coefficients cannot be predicted accurately and heat transfer surfaces get fouled. This is not much of a problem. The operator can make test runs and repeat them at regular time intervals to determine $U$ and fouling as a function of operating conditions. Further we assumed that the inlet temperature $T_{0}$ equals $T_{c}$. This can be achieved by filling the first part of the tube with an inert, heat conducting material, so that the reaction mixture is heated up before entering the catalyst bed.

More difficult are uncertainties in kinetics or changes in kinetics, e.g. due to catalyst aging or fouling. The prediction of the exact value of the criterion depends on the accuracy of the kinetics, that is on $p$ and $\gamma_{p}$. If catalyst aging affects both reactions in equal way, $T_{R}$ is not affected and only $k_{R}$ is lowered, which requires a longer tube to achieve optimum results. However, if $p$ and $\gamma_{p}$ are also affected - that is the activation energies-also $T_{m a}$ is influenced. This requires ad- ditional test runs to determine how the required value of $T_{\text {ma }}$ is determined by catalyst behaviour. These effects all ask for an overdesign of the reactor tube length and the operation of the reactor with lower coolant temperatures in the initial period, when the catalyst is still very active.

Often kinetics are unknown so that a reactor tube bundle has been designed empirically. In this case $T_{m a}$ has to be determined empirically in an isothermal experimental reactor and in a separate simulation in a single reactor tube with the same diameter as in the industrial reactor, the required values of the dimensionless group can also be determined empirically. Once these have been established the reactor can be operated according to the criterion.

Hot spots always will occur in tubular reactors. They are not dangerous as long as the reactor is operated in the regime of low parametric sensitivity. Our studies reveal that hot spots always will be found in the first part of the reactor tube. From experiments or calculations can be determined where at a certain load the hot spot will be located approximately. From the reactor entrance up to this location thermocouples can be placed at regular intervals to determine temperature gradients. For safe operation these gradients have to decrease in the direction of the hot spot.

Finally we like to mention that -for the basic design given in this paper of an inside tube diameter of 24 $\times 10^{-3} \mathrm{~m}$ and $T_{c}=643 \mathrm{~K}$ in order to maintain a high yield-we have checked the criterion of van Welsenaere and Froment [1].

At these conditions their criterion would give a maximum tube diameter of $35 \times 10^{-3} \mathrm{~m}$, whereas runaway is actually found at $32 \times 10^{-3} \mathrm{~m}$. The overestimate is due to the second reaction, which cannot be accounted for with their method.

\section{CONCLUSIONS}

Based on the model presented here, for two consecutive first order exothermic reactions in a cooled tubular pseudo-homogeneous reactor we may conclude that:

- the method outlined here requires kinetic information on both the desired and the undesired reaction

- the objective to obtain high reactor yields requires more stringent design and operating conditions than the prevention of reactor temperature runaway

-the reactor length required to stop the reaction at the point of maximum yield can only be obtained by numerical integration

-the criterion derived and the corresponding requirements (20), (23) and/or (29) enable us to select a combination of design of reactor parameters to achieve a desired yield.

Acknowledgement-The authors wish to acknowledge the assistance of Dr. Ptasinsky who studied the behaviour of the locus of maxima surface in the three-dimensional space. 


\section{NOTATION}

C concentration of species, $\mathrm{kmol} / \mathrm{m}^{3}$

$c_{p} \quad$ specific heat of reaction mixture, $J / \mathbf{k g ~ K}$

$d_{t}$ tube diameter, $\mathrm{m}$

$E$ activation energy, $\mathrm{J} / \mathbf{k m o l}$

$H \quad \Delta H_{X} / \Delta H_{A}$

$\Delta H \quad$ heat of reaction, $\mathrm{J} / \mathrm{kmol}$ (exothermic)

$k$ reaction velocity constant, $\mathrm{m}^{3} / \mathrm{kg} \mathrm{s}$

$k_{R} \quad$ reference reaction velocity constant, $\mathrm{m}^{3} / \mathrm{kg} \mathrm{s}$

$L$ reactor length, $m$

$p \quad E_{X} / E_{P}$

$p_{\text {tot }} \quad$ reactor pressure, $\mathbf{P a}$

$R$ gas constant, $8.314 \mathrm{~kJ} / \mathrm{kmol} \mathrm{K}$

$R_{w} \quad$ rate of production of species per unit mass of catalyst, $\mathrm{kmol} / \mathrm{kgs}$

$T$ temperature, $\mathrm{K}$

$T_{R} \quad$ reference temperature, at which $k_{R}=k_{X}$ $=k_{P}, \mathbf{K}$

$u \quad$ superficial gas velocity, $\mathrm{m} / \mathrm{s}$

$U$ total heat transfer coefficient, $W / m^{2} K$

$U^{*} \quad 4 U / k_{R} \rho_{B} \rho_{\theta} c_{p} d_{t}$

' $X \quad$ relative degree of conversion

$z \quad$ coordinate in the direction of flow, $m$

$Z \quad z / L$, dimensionless reactor length

$D a \quad k_{R} \rho_{B} L / u$, dimensionless residence time

Greek symbols

$\gamma_{P} \quad E_{P} / R T_{R}$, dimensionless activation energy

$\theta_{P} \quad$ reactor yield

$\left(\theta_{P}\right)_{\text {opt }}$ isothermal maximum reactor yield

$\kappa\left(k_{P} / k_{R}\right.$, dimensionless reaction velocity constant for the desired reaction
$\rho_{B} \quad$ bulk catalyst density, $\mathrm{kg} / \mathrm{m}^{3}$

$\rho_{g}$ density of reaction mixture, $\mathrm{kg} / \mathrm{m}^{3}$

$\tau \quad T / T_{R}$, dimensionless temperature

$\Delta \tau_{\text {ad }} \quad \Delta H_{A} C_{A 0} / T_{R} \rho_{g} c_{p}$

\section{Subscripts}

$\begin{array}{ll}A & \text { reactant } \\ c & \text { coolant } \\ \text { opt } & \text { optimum } \\ m & \text { maximum or hot spot } \\ m a & \text { maximum allowable } \\ P & \text { desired product } \\ X & \text { undesired product } \\ 0 & \text { inlet }\end{array}$

\section{REFERENCES}

[1] Westerterp K. R. and Ptasinsky K. J., Chem. Engng Sci. 198439235.

[2] Westerterp K. R. and Ptasinsky K. J., Chem. Engng Sci. 198439244.

[3] Van Welsenaere R. J. and Froment G. F., Chem. Engng Sci. 1970251503.

[4] De Maria F., Longfield J. E. and Butler G., Ind. Engng Chem. 196153259.

[5] Westerterp K. R., Chem. Engng Sci. 196217423.

[6] Carberry J. J. and White D., Ind. Engng Chem. 196961 (7) 27.

[7] Kirk-Othmer, Encyclopedia of Chemical Technology, Vol. 12, p. 129. Wiley, London (1980).

[8] Westerterp K. R., van Swaaij W. P. M. and Beenackers A. A. C. M., Chemical Reactor Design and Operation. Wiley, London (1984).

[9] Wiedemann O. and Gierer W., Chem. Engng 19798662. 